\title{
Epidemiologie und arbeitsmedizinische Aspekte des Pleuramesothelioms
}

\section{Epidemiological and Occupational Medicine Aspects of Pleural Mesothelioma}

Autoren

Institute
B. Karabin-Kehl' , V. Harth ${ }^{1}$, A. M. Preisser ${ }^{2}$

'Institut und Hochschulambulanz für Arbeitsmedizin, Umweltmedizin und Public Health (IAUP) und Präventivmedizinisches Zentrum für arbeits- und umweltbedingte Erkrankungen (Institutsleiter: Univ.-Prof. Dr. med. Volker Harth, MPH)

Zentralinstitut für Arbeitsmedizin und Maritime Medizin, Universitätsklinikum Hamburg-Eppendorf (Institutsleiterin (komm.): Prof. Dr. med. Ingrid Moll) eingereicht 20.11.2012 akzeptiert nach Revision 6. 12.2012

\section{Bibliografie}

Dol http://dx.doi.org/ 10.1055/s-0032-1326061 Online-Publikation: 17.1.2013 Pneumologie 2013; 67: 209-218 (c) Georg Thieme Verlag KG Stuttgart · New York ISSN 0934-8387

\section{Korrespondenzadresse} Dr. med. Alexandra M. Preisser Universitätsklinikum Hamburg-Eppendorf Zentralinstitut für Arbeitsmedizin und Maritime Medizin Seewartenstraße 10 20459 Hamburg a.preisser@uke.de

\section{Serienherausgeber}

C. Grohé, Berlin

\section{Zusammenfassung $\nabla$}

Das maligne Mesotheliom der Pleura gilt als Signaltumor für eine (berufliche) Asbestexposition. Aufgrund der langen Latenzzeit zwischen der Erstexposition gegenüber Asbestfasern und dem Auftreten des Tumors treten fast 20 Jahre nach dem Asbestverbot in Deutschland weiterhin Erkrankungsfälle auf. Von besonderem Interesse ist dabei die epidemiologische Entwicklung dieser asbestbedingten Erkrankung. In Deutschland wird aktuell diskutiert, ob die Inzidenz des malignen Pleuramesothelioms weiterhin ansteigt, ob sie bereits ein Plateau erreicht hat oder ob sie in den nächsten Jahren sinken wird. Der Verlauf der Inzidenz wird dabei wesentlich von der früheren Verbrauchsmenge, den Verwendungsgebieten und der schrittweisen Substitution von Asbest bestimmt. Die Prävention asbestbedingter $\mathrm{Er}$ krankungen stellt aufgrund historischer Expositionen, aber auch möglicher Expositionen durch die Sanierung vorhandener Altlasten ein vordringliches Thema in der Arbeitsmedizin dar. Ziel dabei ist es, einen wirkungsvollen Arbeitsschutz zu gewährleisten und asbestexponierte Arbeitnehmer - auch über ihr Tätigkeitsende hinaus in möglichst effektiven und effizienten Früherkennungsprogrammen zu betreuen. Neue Technologien, wie die Nanotechnologie mit Carbonnanotubes, können darüber hinaus neue potenzielle Gesundheitsgefahren beinhalten.

\section{Einleitung}

\section{$\nabla$}

Der berufliche Kontakt zu Asbest gilt als Hauptursache in der Entstehung des malignen Pleuramesothelioms (MPM). Im Jahre 1993 wurde in Deutschland die Herstellung und Verwendung von Asbest verboten ( $\S 15$ GefStoffV [1]). Jedoch wird auch heute noch in vielen Ländern der Welt Asbest abgebaut und verarbeitet. 2010 wurden

\section{Abstract \\ $\nabla$}

Malignant mesothelioma of the pleura represents a signal tumour for (occupational) exposure to asbestos. Almost 20 years after the ban of asbestos in Germany, incident cases are still occurring due to the long latency period between the initial exposure to asbestos and the onset of the tumour. Of particular interest is the development of mesothelioma epidemiology. In Germany, it is extensively discussed whether the incidence of malignant pleura mesothelioma continues to rise, has already reached a plateau or is expected to decline in the next few years. The development is predominantly caused by the total asbestos use, its application and the gradual substitution of asbestos. The prevention of asbestos-related diseases due to former exposures, but also due to existing asbestos contaminations and their restoration is still a hot topic in occupational medicine. It is thus of major importance to ensure an adequate occupational safety and to care for asbestos-exposed workers - even after cessation of their exposure - with effective and efficient measures of early detection. New technologies, such as nanotechnology with carbon nanotubes, represent new potential health hazards.

weltweit 2,01 Mio. Tonnen Asbest produziert ( Tab.1) [2].

Aufgrund der langjährigen baulichen Nutzung sind in Deutschland noch viele Asbestaltlasten zu berücksichtigen, welche vor allem bei Abriss- und Sanierungsarbeiten zu Asbestexpositionen geführt haben und weiterhin führen. Aufgrund der oft mehrere Jahrzehnte dauernden Latenz bis zum Auftreten der Erkrankung steigt die Inzidenz 
Tab. 1 Übersicht der Asbestproduktion einzelner Länder und weltweit in Tonnen [2].

\begin{tabular}{|lrrrrr}
\hline Asbest: Weltproduktion im Ländervergleich (Angaben in Tonnen) & & & \\
Country & $\mathbf{2 0 0 6}$ & $\mathbf{2 0 0 7}$ & $\mathbf{2 0 0 8}$ & $\mathbf{2 0 0 9}$ & $\mathbf{2 0 1 0}$ \\
\hline Brasilien & 227.000 & 254.000 & 288.000 & 288.000 & 270.000 \\
\hline Kanada & 200.000 & 180.000 & 160.000 & 150.000 & 100.000 \\
\hline China & 360.000 & 390.000 & 380.000 & 380.000 & 19.000 \\
\hline Indien & 20.000 & 21.000 & 20.000 & 230.000 \\
\hline Kasachstan & 315.000 & 293.000 & 230.000 & 1.000 .000 \\
\hline Russland & 925.000 & 1.030 .000 & 1.020 .000 & 5.300 & 1.000 .000 \\
\hline Sonstige & 106.099 & 84.780 & 11.800 & 1.300 \\
\hline Total & $\mathbf{2 . 1 5 0 . 0 0 0}$ & $\mathbf{2 . 2 5 0 . 0 0 0}$ & $\mathbf{2 . 1 1 0 . 0 0 0}$ & $\mathbf{2 . 1 3 0 . 0 0 0}$ & $\mathbf{2 . 0 1 0 . 0 0 0}$
\end{tabular}

der asbestbedingten malignen Tumore, wie das Pleuramesotheliom, weiterhin an. Der Häufigkeitsgipfel der asbestbedingten Tumore, insbesondere des Pleuramesothelioms, wird in Europa zwischen 2010 und 2020 erwartet [3].

Neben Asbestfasern in der Arbeits- und Umwelt stehen inzwischen auch neue Materialien wie Nanoröhren (sogenannte Tubes) wegen möglicher asbestfaserähnlicher Eigenschaften im Fokus der wissenschaftlichen Diskussion. Auch sie stehen im Verdacht, bei der Pathogenese von Mesotheliomen zukünftig eine Rolle spielen zu können [4].

\section{Klinik}

$\nabla$

Die Symptomatik des malignen Pleuramesothelioms ist anfangs unspezifisch und beginnt meist mit Müdigkeit, Abgeschlagenheit sowie Dyspnoe. Diese kann entweder neu auftreten oder bestehende Luftnot anderer Ursache verschlimmern. Des Weiteren sind Husten und thorakale Schmerzen typisch. Sie entstehen meist durch die Infiltration des Tumors in die sensibel innervierte Pleura. Die Entzündung und Reibung der beiden Pleurablätter ist hiervon Folge und führt zur weiteren Schmerzverstärkung. Beim Fortschreiten der Erkrankung kann es durch das Wachstum des Tumors zu einer Schrumpfung der betroffenen Thoraxseite und zu einer Verdrängung der Lunge kommen. Auch ein einseitiger Pleuraerguss, der die Dyspnoesymptomatik weiter verschlimmert, ist möglich. Neben einer B-Symptomatik mit Gewichtsverlust, Nachtschweiß und Fieber kann eine vermehrte Sputumproduktion beobachtet werden $[5,6]$.

\section{Pathogenese}

Oberdörster et al. [7] sowie Miserocchi et al. [8] führten tierexperimentelle morphologische Untersuchungen zum Transport der Asbestfasern ins Lungeninterstitium und schließlich in den Pleuralraum durch. Sie beschrieben, dass die Fasern entweder parazellulär ober über verletzte Epithelzellen ins Interstitium gelangen. Von dort erfolgt entweder der Transport der Asbestfasern per Lymphflüssigkeit oder Blutstrom oder diese gelangen passiv in den Interpleuralraum. Aufgrund der unterschiedlichen Anatomie vom Menschen zum Nagetier ist eine Übertragung dieser Befunde auf den Menschen jedoch nur eingeschränkt möglich. Neue Techniken, wie die Kernspinresonanzspektroskopie (NMR) und Multiphotonenmikroskopie, ermöglichen es, detaillierte Erkenntnisse über die intrapulmonalen Transportwege von Asbest im Tierversuch erzielen zu können.
Tab.2 Mesotheliom-Todesfälle und vorhergesagte Todeszahlen [3, 36].

\begin{tabular}{|lrr|} 
& 1994 bis $\mathbf{2 0 0 8}$ & $\mathbf{2 0 1 5}$ bis $\mathbf{2 0 1 9}$ \\
\hline Großbritannien & 13.517 & 8.750 \\
\hline Frankreich & 6.608 & 7.750 \\
\hline Deutschland & 9.569 & 6.850 \\
\hline Italien & 3.706 & 4.760 \\
Niederlande & 5.141 & 4.650 \\
\hline Total & $\mathbf{3 8 . 5 4 1}$ & $\mathbf{3 2 . 7 6 0}$
\end{tabular}

Aktuell wird erforscht, wie Asbestfasern pleurale Mesothelzellen auf direktem oder indirektem Wege über Makrophagen schädigen können. Die zentrale Hypothese der Mesotheliomentstehung beinhaltet hierbei folgende Kausalkette:

- Verminderte Clearence und nachfolgende Entzündungsreaktion

- Zellrecruitment und Aktivierung, später Fibrosierung

- Entstehung freier Radikale (ROS, RNS), Chemokine, Cytokine und Wachstumsfaktoren

- DNA-Schäden, veränderte Mitoserate, Apoptose, Nekrose

- Aktivierung zellulärer Signalwege, Zell-Proliferation, gestörte Apoptose, fehlerhafte DNA-Reparatur, Inaktivierung von Tumor-Supressor-Genen sowie Aktivierung von Onkogenen [9]. Von amphibolen Asbestarten ist bekannt, dass sie auf ihrer Oberfläche redoxaktive Eisenione enthalten, die Sauerstoffradikale generieren, welche zusätzlich zu Protein- und DNA-Schäden führen [10].

\section{Histologie}

Die malignen mesotheliomen Tumore der Pleura werden entsprechend des führenden histologischen Typs eingeteilt nach

- epitheloid (häufigster Typ)

> sarkomatoid

- biphasisch

- desmoplastisch

- lokalisiertes malignes Mesotheliom [11]

Nach wie vor ist die Unterscheidung zum Adenokarzinom der Lunge pathologisch schwierig. Nur mit genauer immunhistochemischer Untersuchung kann die Differenzialdiagnose gestellt werden. Der Nachweis von Cytokeratin und Vimentin sowie des Markers WT1 (Wilms-Tumor 1) sind typisch für das Pleuramesotheliom, aber negativ beim Adenokarzinom. Umgekehrt verhält es sich mit den Markern CEA, Len $\mathrm{M}_{1}$, Ber EB4, AUA1 und TTF-1, welche beim Pleuramesotheliom nicht nachweisbar sind, jedoch beim Adenokarzinom zu einer positiven Reaktion führen $[6,12]$. 


\begin{tabular}{|c|c|}
\hline Tx & Primärtumor nicht beurteilbar \\
\hline TO & Kein Anhalt für Primärtumor \\
\hline T1 & Tumor befällt ipsilaterale parietale Pleura, mit oder ohne fokale Beteiligung der viszeralen Pleura \\
\hline T1a & Tumor begrenzt auf ipsilaterale parietale (mediastinale, diaphragmale) Pleura. Keine Beteiligung der viszeralen Pleura. \\
\hline T1b & Tumor befällt ipsilaterale parietale (mediastinale, diaphragmale) Pleura. Fokale Beteiligung der viszeralen Pleura. \\
\hline T2 & $\begin{array}{l}\text { Tumor befällt die ipsilaterale Pleuraoberfläche mit wenigstens einem der folgenden Merkmale: } \\
\text { - Konfluierender Tumor der viszeralen Pleura (einschließlich der Fissuren) } \\
\text { - Infiltration der Zwerchfellmuskulatur } \\
\text { - Infiltration des Lungenparenchyms }\end{array}$ \\
\hline T3 & $\begin{array}{l}\text { Tumor befällt die ipsilaterale Pleuraoberfläche mit wenigstens einem der folgenden Merkmale: } \\
\text { - Infiltration der endothorakalen Faszie } \\
\text { - Infiltration von mediastinalem Fettgewebe } \\
\text { - Einzelner Tumorherd mit Infiltration des Weichgewebes der Thoraxwand } \\
\text { - Nicht-transmurale Infiltration des Perikard }\end{array}$ \\
\hline T4 & $\begin{array}{l}\text { Tumor befällt die ipsilaterale Pleuraoberfläche mit wenigstens einem der folgenden Merkmale: } \\
\text { - Diffuse oder multifokale Infiltration der Weichgewebe der Thoraxwand } \\
\text { - Infiltration der Rippe(n) } \\
\text { - Infiltration durch das Zwerchfell in das Peritoneum } \\
\text { - Infiltration anderer Mediastinalorgane } \\
\text { - Direkte Ausbreitung in die kontralaterale Pleura } \\
\text { - Infiltration der Wirbelsäule } \\
\text { - Ausbreitung auf die innere Oberfläche des Perikards } \\
\text { - Perikarderguss mit positiver Zytologie } \\
\text { - Infiltration des Myokards } \\
\text { - Infiltration des Plexus brachialis }\end{array}$ \\
\hline $\mathrm{Nx}$ & Regionäre Lymphknoten nicht beurteilbar \\
\hline NO & Keine regionären Lymphknotenmetastasen \\
\hline N1 & Metastase(n) in ipsilateralen bronchopulmonalen und/oder in ipsilateralen Hiluslymphknoten \\
\hline N2 & $\begin{array}{l}\text { Metastase }(n) \text { in subcarinalen Lymphknoten und/oder ipsilateralen Lymphknoten entlang der Arteria mammaria interna oder in mediastinalen } \\
\text { Lymphknoten }\end{array}$ \\
\hline N3 & $\begin{array}{l}\text { Metastase(n) in kontralateralen mediastinalen Lymphknoten, solchen entlang der Arteria mammaria interna, kontralateralen Hilus- } \\
\text { und/oder ipsi- oder kontralateralen Skalenus- oder supraklavikulären Lymphknoten }\end{array}$ \\
\hline Mx & Fernmetastasen nicht beurteilbar \\
\hline MO & Fernmetastasen nicht nachweisbar \\
\hline M1 & Fernmetastase(n) \\
\hline
\end{tabular}

\section{Prognostische Faktoren}

Das Pleuramesotheliom ist ein sehr schnell wachsender Tumor, die mittlere Überlebenszeit nach Diagnosestellung der Erkrankung beträgt 7 bis 9 Monate ohne Therapie [13]. Von prognostischem Wert scheint dabei der histologische Subtyp zu sein: Der nicht-epitheloide Subtyp hat eine schlechtere Prognose mit einer mittleren Überlebenszeit zwischen 8 und 13 Monaten, diese liegt für den epitheloiden Subtyp bei 19 Monaten [14,15]. Auch auf der Inflammation basierende Indices wie der mGPS (modified Glasgow Prognostic Score) und die NLR (Neutrophil Lymphocyte Ratio) korrelieren mit anhaltender Neoangiogenese sowie erhöhter Zellproliferation und somit schnellerem Tumorwachstum [16].

\section{Diagnostik und Staging}

Beim Auftreten von Symptomen kann die angefertigte Thoraxröntgenaufnahme einen Pleuraerguss oder eine pleurale Verdickung mit Pleuraplaques aufweisen [6]. Die Computertomografie (CT) ist das meist gebrauchte diagnostische Instrument, um den Verdacht auf ein MPM zu bestätigen. Im Frühstadium erscheint der Tumor fokal mit einer Pleuraverdickung und/oder mit einem Pleuraerguss. In späteren Stadien erreicht der Tumor eine große Masse, die die Lunge regelrecht umschließen kann [17]. Um den Verdacht auf ein Pleuramesotheliom zu sichern, sollte eine Biop- sie, wenn möglich, mittels Thorakoskopie, durchgeführt werden. Die Zytologie aus dem Pleurapunktat ist aufgrund der hohen diagnostischen Fehlerrate alleine nicht ausreichend. Wenn die Thorakoskopie technisch oder aufgrund von Kontraindikationen nicht möglich ist, kann eine Feinnadelpunktion zum Ziel führen. Aufgrund der schwierigen Differentialdiagnostik ist eine immunhistologische Untersuchung des Biopsates unabdingbar [18]. In der Diagnosestellung und auch für das weitere Staging zeigten sich in Studien zahlreiche Vorteile in der Verwendung der Positronen-Emissions-Tomografie mit Fluordesoxyglucose (FDGPET) [19]. Die Wertigkeit der PET-Computertomografie und weiterer Staging-Untersuchungen wie der endosonografischen Lymphknotenpunktion (EBUS-FNA) ist jedoch noch nicht abschließend zu bewerten [5]. Für das Staging des malignen Pleuramesothelioms sind verschiedene Tumor-Klassifizierungen bekannt, die größte klinische Relevanz besitzt die TNM-Klassifikation der „Union internationale contre le cancer“ (UICC) ( Tab.3 und Tab.4). Dieses System wird aufgrund der fraglichen Prognose-Abschätzung kontrovers diskutiert. Von der International Association for the Study of Lung Cancer Staging Committee wurden aktuell 3101 Patientendaten ausgewertet. Es konnte gezeigt werden, dass durch Erweiterung und Neugestaltung des StagingSystems bessere Korrelationen zwischen dem Staging-Ergebnis und der Prognose erreicht werden können. Diese Ergebnisse werden auch durch die Auswertung früherer kleinerer Datenbestände unterstützt und beweisen damit die Notwendigkeit der Überarbeitung $[15,20]$. 
Tab. 4 Stadien nach UICC (Union Internationale Contre le Cancer) [58]

\begin{tabular}{llll|}
\hline Stadium IA & T1a & N0 & M0 \\
\hline Stadium IB & T1b & N0 & M0 \\
\hline Stadium II & T2 & N0 & M0 \\
\hline Stadium III & T1, T2 & N1 & M0 \\
& T1, T2 & N2 & M0 \\
& T3 & N0, N1, N2 & M0 \\
\hline Stadium IV & T4 & Jedes N & M0 \\
& Jedes T & N3 & M0 \\
& Jedes T & Jedes N & M1 \\
\hline
\end{tabular}

\section{Asbest}

\section{$\nabla$}

Der Begriff Asbest ist eine Sammelbezeichnung für natürlich vorkommende Silikat-Minerale, die in Serpentine und Amphibole eingeteilt werden. Mit ca. 90 bis 95\% Anteil gehört Chrysotil (Weißasbest) weltweit zu der am häufigsten industriell verwendeten Asbestart. Es wird den Serpentinen zugeordnet und erscheint elektronenoptisch spiralig. Nur 5 bis $10 \%$ der weltweit verwendeten Asbestmenge sind Amphibole, zu denen Krokydolith (sog. Blauasbest, ein Natriumeisensilikat), Amosit (sog. Braunasbest), Aktinolith, Tremolith und Anthophyllit gehören ( $\bullet$ Abb.1). Sie besitzen eine nadelförmige Struktur und sind besonders gut alveolengängig [21].

Asbest wird zumeist im Tagebau gefördert. Die weltweite Jahresförderung beträgt etwa 2 Mio. Tonnen, das Hauptabbauland ist seit Jahren Russland mit einer Produktion von etwa 1 Mio. t Asbest (2010), gefolgt von China mit 400.000 t. Weitere wichtige Abbauländer sind - in der Reihenfolge der Fördermengen - Brasilien, Kasachstan, Kanada, Indien, Simbabwe und Argentinien ( Tab. 1) [2].

Aufgrund der vielfältigen Eigenschaften von Asbest (griechisch asbestos: nicht brennbar, unzerstörbar) wurde es als „Mineral der 1.000 Möglichkeiten“ bezeichnet und fand in Deutschland in über 3.000 verschiedenen Anwendungsbereichen von Industrie und Handwerk Verwendung. Besondere Eigenschaften von Asbest sind u.a. Hitzebeständigkeit, Elastizität, Zugfähigkeit, Beständigkeit gegenüber Chemikalien sowie hohe Isolierfähigkeit. Die Fasern können versponnen und somit zu verschiedenen Textilien verarbeitet werden. Die Nutzungsmöglichkeiten sind vielseitig wie bei kaum einer anderen Substanz. Vor allem in der Bauwirtschaft wurde Asbest im Zement (z.B. früher in Spritzze- ment oder Faserzementplatten), als Isolierungsmaterial, in Dichtungen, als Dachziegel, in Feuerschutzeinrichtungen und vielem mehr verwendet. Auch im Elektrizitätswesen, der Schiffs- und Automobilindustrie sowie im Handwerks-, Haushalts- und Hobbybereich wurden asbesthaltige Produkte wie z.B. Bremsen, Kupplungen, Textilprodukte (Garne, Bänder, Schnüre etc.) eingesetzt. Häufig war Asbest auch Bestandteil von persönlicher Schutzausrüstung der Arbeiter (z.B. Handschuhe, Decken, Schutzanzüge). Selbst die Filter mancher Atemmasken bestanden z.T. aus Asbest.

Entsprechend den Einsatzgebieten sind auch die Berufsfelder mit Asbestexposition sehr zahlreich. Im BK-Report 1/2007 [22] sind allein 60 Berufsgruppen, die eine Vielzahl von Einzelberufen umfassen, aufgeführt. Besonders gefährdet sind u.a. Chemiearbeiter, Isolierer, Schlosser, Dachdecker, Maurer, Sanitär- und Elektroinstallateure, Schweißer, Brennschneider und Maschinenschlosser. Um eine mögliche Asbestexposition zu eruieren, ist daher eine genaue Arbeitsanamnese unabdingbar.

Besonders beim Abbau von Asbesterzen und deren Weiterverarbeitung treten hohe Expositionen auf. Bei bisher durchgeführten Messungen an Arbeitsplätzen in Steinbrüchen wurden Asbestfaserkonzentrationen von bis zu 0,4 Mio. Fasern/m $/ \mathrm{m}^{3}$ Luft $(90 \%$ Wert) erreicht. Noch höhere Werte finden sich bei der Aufbereitung von Chrysotil. Bei der Spritzasbesttechnologie wurden 30 bis 300 Mio. biobeständige Asbestfasern $/ \mathrm{m}^{3}$ Luft gemessen. Besonders gefährlich ist das Zerkleinern, Brechen, Sägen, Bohren, und Schleifen von Asbest, weil hierbei reichlich Staub ensteht und Asbeststaubpartikel eingeatmet werden können. Die Exposition kann sich durch die Tätigkeiten in schlecht belüfteten Räumen weiter erhöhen, wie dies besonders im Schiffbau und Waggonbau bei Installation und Wartungsarbeiten gegeben war. Aber nicht nur die Arbeiter, die direkt mit Asbest Umgang hatten, sondern auch sogenannte „By-Stander“ an nahegelegenen Arbeitsplätzen oder Familienmitglieder, die die Arbeitskleidung reinigten, können belastet sein. Sie waren den Stäuben häufig ungeschützt ausgesetzt und oftmals über die möglichen Expositionen nicht unterrichtet $[23,24]$.

Seit dem Asbest-Verbot sind aktuell in Deutschland die Abbruchund Sanierungsarbeiten die Hauptursache für eine mögliche Asbestexposition. Bei der Sanierung alter Gebäude und dem Austausch asbesthaltiger Produkte müssen die Arbeiter besondere Schutzmaßnahmen einhalten. Dazu gehören Expositionsminimierung durch Atemschutz und strikte Trennung von Asbest-

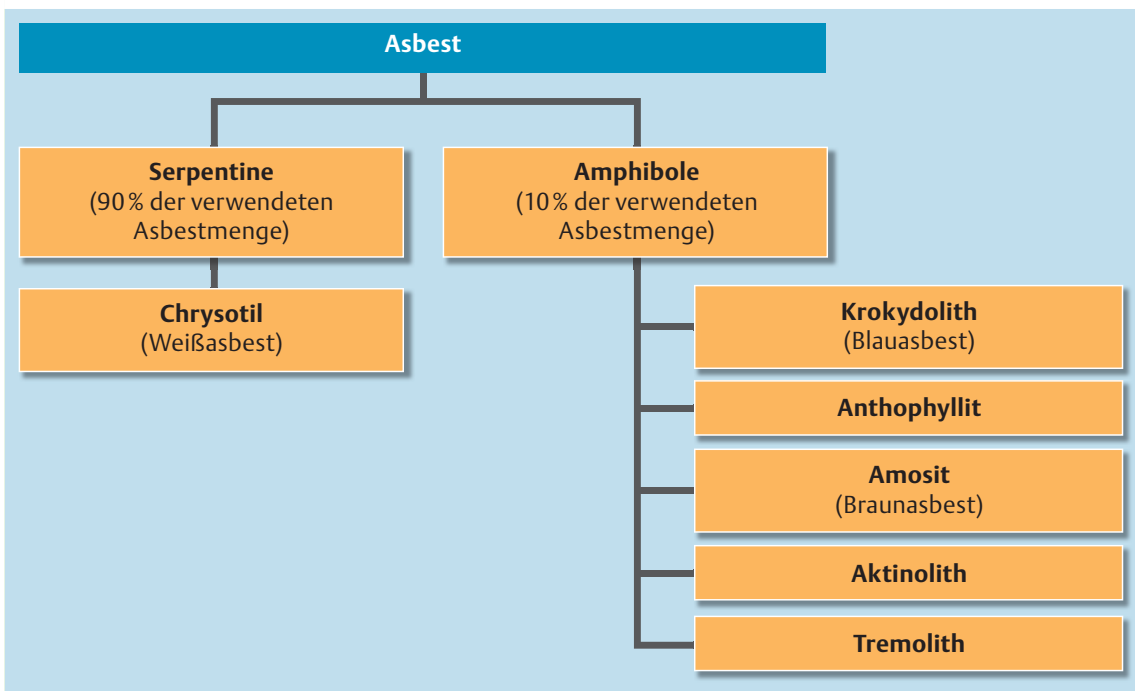

Abb.1 Einteilung der Asbestgruppen 


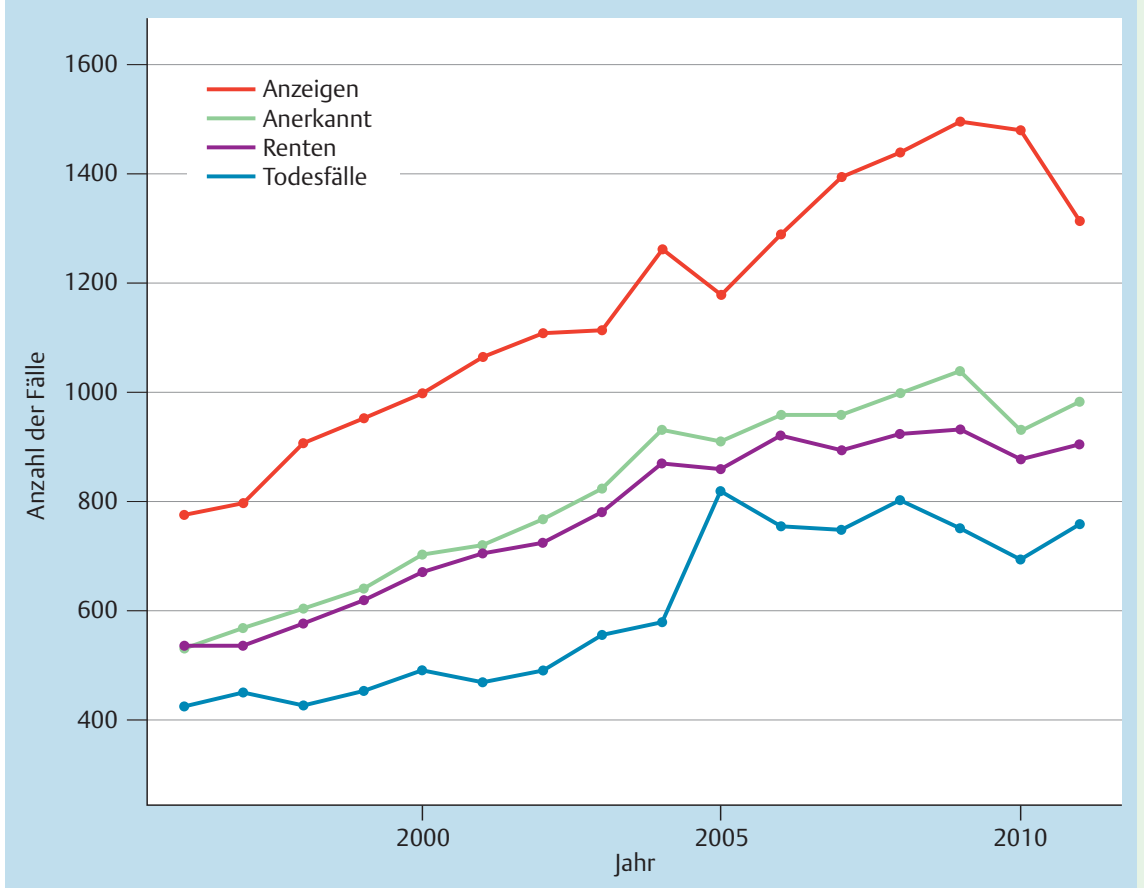

Abb.2 Verdachtsanzeigen, Anerkennungen, Rentenfälle und Todesfälle der BK 4105 („Durch Asbest verursachtes Mesotheliom des Rippenfells, Bauchfells oder des Perikards“) zwischen 1996 und 2011, nach [31].

Schutzkleidung und sonstiger Arbeitskleidung. Behälter mit Asbest und asbestverunreinigten Materialien bedürfen einer speziellen Kennzeichnung [25].

Neben der beruflichen Exposition und dem natürlichen AsbestVorkommen ist eine Exposition durch viele asbesthaltige Mineralien, wie z.B. Speckstein (Talkum) und Erionit, möglich. Auch durch Verwitterung (ca. 100t/Jahr) und Abrieb von Straßendecken und Bremsbelägen (unter $17 \mathrm{t} / \mathrm{Jahr}$ ) ergeben sich relevante Belastungen von ca. 100 bis maximal 330 Fasern/m³ Luft [26].

\section{Epidemiologie \\ $\nabla$}

Der Zusammenhang zwischen dem Umgang mit Asbest und dem Auftreten maligner Tumore der Pleura und des Peritoneums wurde bereits von Wagner et al. im Jahre 1960 belegt [27]. Seither konnte in verschiedenen tierexperimentellen und epidemiologischen Studien die Kanzerogenität von Asbest und die asbestbedingte Verursachung des malignen Mesothelioms bestätigt werden. Dabei wurde intensiv diskutiert, ob nur ambiphole Asbestmaterialien oder auch das Chrysotil Mesotheliome verursachen kann. Zwischenzeitlich wird davon ausgegangen, dass Chrysotil mit seiner spiralförmigen elektronenoptischen Erscheinung eine geringere kanzerogene Potenz als die Amphibol-Asbeste besitzt. Jedoch sind zahlreiche Fälle von malignen Mesotheliomen dokumentiert, bei denen die Verursachung durch Chrysotil wahrscheinlich ist. Es ist davon auszugehen, dass in vielen Industrien wie Bergbau, Handwerk und ähnlichen vermutlich kein reines Chrysotil, sondern Mischfasern zum Einsatz kamen [28].

Lenters et al. [29] zeigten in einer aktuellen Metaanalyse, dass die Beurteilung der Kanzerogenität des Chrysotils im Vergleich zu den Amphibolen schwierig ist, da viele der untersuchten Studien erhebliche Einschränkungen in der Beurteilung der Faserkomponente aufwiesen. Festzuhalten ist aber, dass Amphibole eine lange Biobeständigkeit besitzen. Chrysotile sind dagegen im Ge-

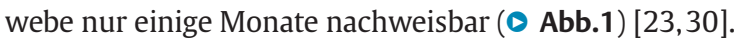

Beruflich bedingte Krebserkrankungen sind für mehr als die Hälfte (ca. 54\%) der berufskrankheitenbedingten Todesfälle ursächlich, wobei die asbestbedingten Tumore hauptursächlich sind [31]. Wurde zu Beginn des Jahrtausends noch davon ausgegangen, dass das Plateau für die Anzahl des asbestbedingten Pleuramesothelioms erreicht sei [32], zeigt nunmehr die Betrachtung der aktuellen Berufskrankheitenstatistik für Deutschland weiterhin einen fast stetigen Anstieg der angezeigten Mesotheliom-Fälle. Kamen im Jahr 1990 noch 467 Verdachtsfälle zur Anzeige, so waren es 2009 bereits 1494 Verdachtsfälle, wobei in demselben Jahr 929 neue Fälle als Berufskrankheit anerkannt wurden. Auch die neuesten Zahlen zeigen - nach einem vorübergehenden Abfall für 2010-für das Jahr 2011 eine Fortsetzung dieses Trends (siehe Abb.2) [31].

Schätzungen zu Inzidenz von Mesotheliomerkrankungen und die Abschätzung zukünftiger Fallzahlen leisten darüber hinaus einen wichtigen Beitrag zur Überprüfung der Wirksamkeit von Früherkennungsmaßnahmen und der Abschätzung entstehender Kosten für das Gesundheitswesen [33]. Europaweit steht zur Diskussion, ob die Inzidenz des Pleuramesothelioms einen weiteren Anstieg zeigt, ein Peak wird hierbei um das Jahr 2018 erwartet [3]. Für Großbritannien publizierten Hodgson et al. [34] eine Prognose für die Inzidenz- und Mortalitätsraten des Mesothelioms. Sie betrachteten hierfür Daten aus dem Zeitraum von 1968 bis 2001. Die Anzahl der Todesfälle stieg dabei rapide von 153 Toten in 1968 auf 1848 Tote in 2001. Das Maximum wurde mit über 2000 Toten für die Jahre 2011 bis 2015 vorhergesagt. Insgesamt schätzen die Autoren, dass bis 2050 kumulativ 90.000 Sterbefälle durch das Mesotheliom in Großbritannien auftreten werden.

In den USA ist das maligne Mesotheliom der Pleura ebenfalls ein vergleichsweise seltener Tumor, jedoch steigen auch hier die Fallzahlen in den letzten Jahrzehnten stetig an [35]. Nach der Auswertung der Daten der "Surveillance, Epidemiology and End Results" (SEER) von 1973 bis 2005 von Price and Ware [33] werden für 2008 bis 2042 insgesamt rund 68.000 Mesotheliomfälle in den USA erwartet, bei denen Asbest aber nur noch in $34 \%$ als Hauptverursacher angesehen werden könne. Die Autoren rech- 
nen hoch, dass ab dem Jahr 2042 Asbest in der Mesotheliomentstehung in den USA keine wesentliche Rolle mehr einnehmen werde.

Weltweit wurden von 1994 bis 2008 (in 83 erfassten Ländern) 92.253 Sterbefälle durch ein Mesotheliom registriert, davon 38.121 mit Pleuramesotheliom. Die fünf Länder mit der höchsten Todeszahl waren die USA, Großbritannien (mit Nordirland), Japan, Deutschland und Frankreich. Die Verteilung Männer zu Frauen betrug dabei 3:1, begründet durch die vermehrte berufliche Exposition der Männer [36]. Eine Übersicht über die weltweit zu erwartenden Mesotheliom-Fälle gibt $\bullet$ Tab. 2 .

Wesentlich ursächlich für die aktuelle Entwicklung der Erkrankungszahlen ist der hohe Asbestverbrauch in den 1970er- und 1980er-Jahren in Deutschland und die durchschnittliche Latenzzeit zwischen Erstexposition und der Diagnosestellung von mehr als 30 Jahren. Weltweit führt dies dazu, dass Männer der Geburtsjahrgänge 1945 bis 1950 die höchste Wahrscheinlichkeit für eine Erkrankung an einem MPM haben $[3,36]$. Ein weiterer zu berücksichtigender Faktor ist die Zunahme der Erkrankungen im höheren Alter als Folge des demografischen Wandels. Lag 1980 das durchschnittliche Sterbealter bei 59,2 Jahren, betrug es 2000 schon 65,1 Jahre, Tendenz steigend. Zzt. liegt das mittlere Sterbealter bei 70 Jahren.

Schwerpunkte besonders gefährdeter Berufe in Bezug auf das MPM im Vergleich zu den Berufen insgesamt mit bestandener Asbesteinwirkung konnten nicht festgestellt werden [32].

Aufgrund der ärztlichen Anzeigepflicht sollte jeder Arzt bereits im Verdachtsfall eine Berufskrankheit bei dem Unfallversicherungsträger (Berufsgenossenschaft oder Unfallkasse) melden. Dies ist auch ohne Zustimmung des Patienten möglich, dieser sollte aber über die Anzeige informiert werden. Das maligne Pleuramesotheliom gilt als Signaltumor für eine meist berufliche Asbestexposition und ist daher nach BKNummer 4105 („Durch Asbest verursachtes Mesotheliom des Rippenfells, des Bauchfells oder Perikards“) anzuzeigen. Dies gilt auch dann, wenn eine mögliche Asbestexposition arbeitsanamnestisch nicht eruierbar ist. Im weiteren Verfahren wird die Exposition retrospektiv ermittelt. Aufgrund der langen Latenzzeit des MPM gestaltet sich dies jedoch häufig als schwierig, da die Betriebe zum Teil nicht mehr existieren [25, 30]. Ebenso wichtig ist daher die Erhebung einer umfangreichen und detaillierten Arbeitsanamnese. Anders als beim Lungen- oder Kehlkopfkrebs in Verbindung mit Asbest (BK-Nummer 4104) sind zur Anerkennung keine Brückenbefunde oder der Nachweis von 25 Asbestfaserjahren erforderlich [11,37].

\section{Weitere diskutierte Ursachen des Pleuramesothelioms $\nabla$}

\section{Genetische Faktoren}

In der Literatur wird von einigen Autoren bei der Entstehung des Pleuramesothelioms ein Zusammenhang mit genetischen Faktoren postuliert. In der Türkei sind Kasuistiken beschrieben, in denen gleich mehrere Generationen von dieser Krankheit betroffen waren. Das Mesotheliom ist dort in einigen Regionen einer der häufigsten Tumoren und Todesursachen bei den Erwachsenen. Ursächlich hierfür ist Erionit, eine Asbestunterart, die in diesen Regionen natürlich im Felsen vorkommt. Aus dem Felsgestein bauten die Einwohner ihre Häuser. Ein solches Dorf ist Karain. In dem Dorf Kalik wird dieselbe Steinart verwendet wie in Karain, jedoch wird hier nur von einem einzigen Mesotheliomfall berichtet, und dies von einer Frau, die aus Karain weggezogen ist. Nähere Betrachtungen der Stammbäume verschiedener Familien der Dörfer konnten bei sechs Familien eine Häufung von Mesotheliomfällen beobachten. Diese führen die Autoren auf eine genetische Beeinflussung im Zusammenhang mit den genannten Umweltfaktoren zurück [6].

\section{Simian-Virus 40}

Das SV40, ein Polyomavirus, kann wie andere Polyomaviren unter bestimmten Voraussetzungen Tumore hervorrufen. Im Labor induzierte das Virus in menschlichen In-vitro-Zellen auch Mesotheliome. In einer Kohortenstudie konnten M. Price et al. [38] in der Personengruppe, die einen mit SV40 kontaminierten Polioimpfstoff erhalten hatte, im Vergleich mit einer Kontrollgruppe jedoch keinen statistisch signifikanten Unterschied bezüglich der Mesotheliomhäufigkeit feststellen.

\section{Strahlung}

Mehrere Fallbeispiele belegen einen Zusammenhang von früherer Strahlentherapie und dem Auftreten von Mesotheliomen ohne anamnestische Asbestexposition [39].

\section{Rauchen}

Es gibt keinen Hinweis, dass Tabakrauch das Risiko der Entwicklung eines Mesothelioms erhöht [6].

\section{Nanotechnologie (Nanomaterialien)}

$\nabla$

Nanomaterialien finden eine immer größere Anwendung in vielen industriellen Einsatzgebieten. So sind Nanopartikel in den vielfältigsten Produkten wie Kleidung, Geschirr, Sanitäranlagen, Reinigungsmittel, Beschichtungsstoffe, Akkus, Filtersysteme und Nahrungsmitteln anzutreffen. In der Medizin werden sie mittlerweile in der Krebstherapie eingesetzt [40]. Dabei bleibt zzt. weitgehend unklar, welchen Weg diese ultrafeinen Strukturen nach der Aufnahme in unserem Körper nehmen oder wie diese weiter verstoffwechselt werden [41].

Aufgrund ihrer einzigartigen physikalischen und chemischen Eigenschaften wecken Carbonnanotubes (CNT) bzw. Multiwalled carbon nanotubes (MWCNTs) besonderes Interesse in Industrie und Forschung [42]. Dabei ähneln Nanokohlenstoffröhren phänotypisch oftmals dem Erscheinungsbild von Asbestfasern. Jaurand et al. [43] konnten in Bezug auf Form, Größe und Reaktivität der Oberfläche gemeinsame Eigenschaften von CNTs und Asbest aufzeigen, verwiesen zugleich aber auch auf die unterschiedliche Struktur und chemische Zusammensetzung. Insgesamt besteht die Hypothese, dass CNTs zur Entstehung der gleichen Zielerkrankungen-insbesondere dem Pleuramesotheliom - beitragen können [44].

Mithilfe von Tierversuchen an Mäusen konnte untersucht werden, inwieweit die Länge der Nanotube-Fasern bei der Gewebeschädigung eine Rolle spielt. Schinwald et al. [45] zeigten, dass die Länge für die akute Inflammation einen wichtigen Faktor darstellt. Besonders Fasern von $4 \mu \mathrm{m}$ Länge weisen eine hohe Pathogenität auf. Verschiedene Untersuchungen an Ratten ließen erkennen, dass Multi Walled (MW)-CNTs Entzündungsreaktionen hervorrufen können. In diesen Studien wurden die CNTs überwiegend intraperitoneal appliziert [46]. Auch Takagi et al. [47, 48] installierten MW-CNTs in die Bauchhöhle von Mäusen. Hier- 
durch konnte tatsächlich die Entstehung von Mesotheliomen nachgewiesen werden. Allerdings wurden in dieser Studie sehr hohe Dosen der Kohlenstoffröhren verwendet, weshalb sie im Bezug auf den Menschen nur eingeschränkt beurteilbar bleibt [48]. Sakamoto et al. [49] konnten bei einer einmaligen intrascrotalen Applikation von MW-CNTs die Entstehung von Mesotheliomen im Tierversuch nachweisen.

Weitere Experimente zeigten, dass CNTs, insbesondere MWCNTs über Veränderungen der intrazellulären Signalwege zu DNA-Schäden und Apoptose führen können [50]. Diese Ergebnisse konnten durch eine neue Untersuchung von Ogasawara Y et al. [51] bestätigt werden. Andere Studien wiederum führten zum Ergebnis, dass Carbonnanotubes zwar Granulome induzieren können, jedoch keine Mesotheliome [52].

Zum jetzigen Zeitpunkt gibt es keine Untersuchung zur Kanzerogenität der Carbonnanotubes unter physiologischen Bedingungen für Menschen. Hinsichtlich ihrer Toxizität konnte jedoch nachgewiesen werden, dass die Hauptmechanismen via oxidativen Stress und Entzündungsreaktionen wirken, welche zu Fibrosen und Granulomen sowie Genschädigungen und schließlich zur Karzinogenität führen können. Humanbasierte Studien zeigen, dass für den Menschen ein gesundheitliches Risiko in der chronischen Inhalation von Carbonnanotubes mit hohen Konzentrationen besteht. Die wichtigsten Zielorgane sind dabei die Atemwege und die Lunge [53].

Inwiefern bestimmte CNTs damit das gleiche gesundheitliche Gefährdungspotenzial wie Asbestfasern aufweisen, kann aufgrund der unterschiedlichen Studiendesigns und -ergebnisse noch nicht abschließend beantwortet werden. Die historischen Erfahrungen und wissenschaftlichen Erkenntnisse zu Asbestfasern sollten Forschungs- und Regulationsgremien dazu anhalten, die potenziellen Gefahren der Nanomaterialien multidisziplinär zu überwachen, um eine effektive Risikokontrolle zu ermöglichen und potenzielle Gesundheitsgefahren möglichst frühzeitig zu entdecken [54].

\section{Arbeitsschutz und Prävention \\ $\nabla$}

Schon vor dem generellen Asbestverbot im Jahre 1993 erfolgte in Deutschland die stufenweise Reduzierung der Verwendung. So wurde 1979 in der BRD in einem Nachtrag zur Unfall-Verhütungs-Vorschrift „Schutz gegen gesundheitsgefährlichen mineralischen Staub“ das Spritzen und Aufsprühen von Asbest verboten. Im Jahre 1982 erfolgte - mit einer 2-jährigen Übergangsfrist - ein Verbot von asbesthaltigen Isoliermaterialien, Spachtelmassen, Anstrichen, Klebstoffen, Mörtel, Boden- und Straßenbelägen. Schließlich wurde im Jahre 1993 der Einsatz von Asbest und asbesthaltigen Produkten sowie deren Herstellung endgültig verboten [25]. Auch Arbeiten an asbesthaltigen Teilen von Gebäuden, Geräten, Maschinen, Anlagen, Fahrzeugen und sonstigen Erzeugnissen sind nicht erlaubt. Ausnahmen bestehen für Abbruch-, Sanierungs- und Instandsetzungsarbeiten oder z.B. für die Chloralkalielektrolyse, wobei asbesthaltige Rohstoffe verwendet werden [55].

Abbruch-, Sanierungs- und Instandsetzungsarbeiten, bei denen eine Asbestexposition auftritt, sind meldepflichtig. Diese dürfen nur mit staubdichter Abtrennung, raumlufttechnischer Anlage, Personenschleuse mit Duschen, kompletter persönlicher Schutzausrüstung, Materialschleuse, Arbeitsplan und Unterweisungen der Mitarbeiter erfolgen.
Als Orientierungswert dienen 15.000 Fasern $/ \mathrm{m}^{3}$; werden diese überschritten, sind entsprechende Schutzmaßnahmen und Vorsorge- sowie nachgehende Untersuchungen zu veranlassen. Die technischen Regeln für Gefahrstoffe im Zusammenhang mit Asbest sind in der TRGS 517: „Tätigkeiten mit potenziell asbesthaltigen mineralischen Rohstoffen“ und die TRGS 519 Asbest: „Abbruch-, Sanierungs- oder Instandhaltungsarbeiten“ [25] festgelegt.

\section{Der besondere Fall}

Beschrieben wird der Fall der Familie G., bestehend aus dem Vater H.G., 76 Jahre, der mit 74 Jahren verstorbenen Ehefrau L.G. und dem mit bereits 42 Jahren verstorbenen Sohn M.G. Der Betriebselektriker Herr H.G. arbeitete über 33 Jahre in einem Kraftwerk, in dem er im Rahmen von Revisionsarbeiten und Reparaturen an Generatoren und Turbinen häufig und z.T. hochgradig gegenüber Asbestfaserstaub exponiert war. Die Ehefrau reinigte zu Hause die asbestverschmutzte Kleidung ihres Ehemannes, der Sohn M.G. wurde in der Phase der häuslichen Kleiderreinigung geboren und war als Säugling bzw. Kleinkind über einen Zeitraum von mindestens 7 Jahren im häuslichen Umfeld der Asbestexposition ausgesetzt.

In den 80er-Jahren traten bei Herrn H.G. erste Symptome wie Atemnot und progrediente Leistungsminderung auf. Aufgrund des Röntgenbefundes und mikroskopisch erkennbarer Asbestkörperchen in der bronchoalveolären Lavage wurde eine Asbestose als Berufserkrankung anerkannt. Wegen eines unklaren Herdbefundes wurde im Jahre 1995 eine linksseitige Lungenoberlappenresektion durchgeführt. Pathologisch fand sich eine Pleuraverschwielung mit älterer Lungenatelektase und dem histologischen Nachweis typischer Asbestkörperchen.

Bei seiner Ehefrau L.G. trat im August 2010 erstmalig ein symptomatischer Pleuraerguss mit der Diagnose eines epitheloiden Pleuramesothelioms rechts auf ( Abb.3). Es erfolgte die Pleurodese und die palliative Chemotherapie mit Carboplatin und Pemetrexed. Wegen einer Tumorprogression wurde im Januar 2011 eine Monochemotherapie mit Vinorelbin eingeleitet.

Der bereits als Kleinkind den Asbeststäuben aus der Kleidung des Vaters gegenüber exponierte Sohn Herr M.G. erkrankte ebenfalls. Zunächst war er als Dipl.-Betriebswirt und Geschäftsführer eines Unternehmens beruflich tätig. Im Jahre 2002 trat bei dem damals 42-jährigen aus völliger Gesundheit heraus und bei negativer Raucheranamnese ein linksseitiger Pleuraerguss auf. Im Rahmen einer posterolateralen Thorakotomie fand sich ein ausgedehnter parietaler und viszeraler Tumor mit Einwachsen von Tumorplaques in die untere Lungenvene, das Mediastinum und den Rezessus (Stadium: pT2 pN2, M0, R2). Histologisch wurde ein Pleuramesotheliom von epitheloiden Typ diagnostiziert. Eine BK-Anzeige wurde erstattet; die BK musste jedoch abgelehnt werden, da aufgrund des außerberuflichen Asbestkontaktes Herr M.G. als Familienangehöriger nicht dem Schutz der gesetzlichen Unfallversicherung unterlag. Herr M.G. verstarb im Alter von 42 Jahren an den Folgen der Erkrankung. 

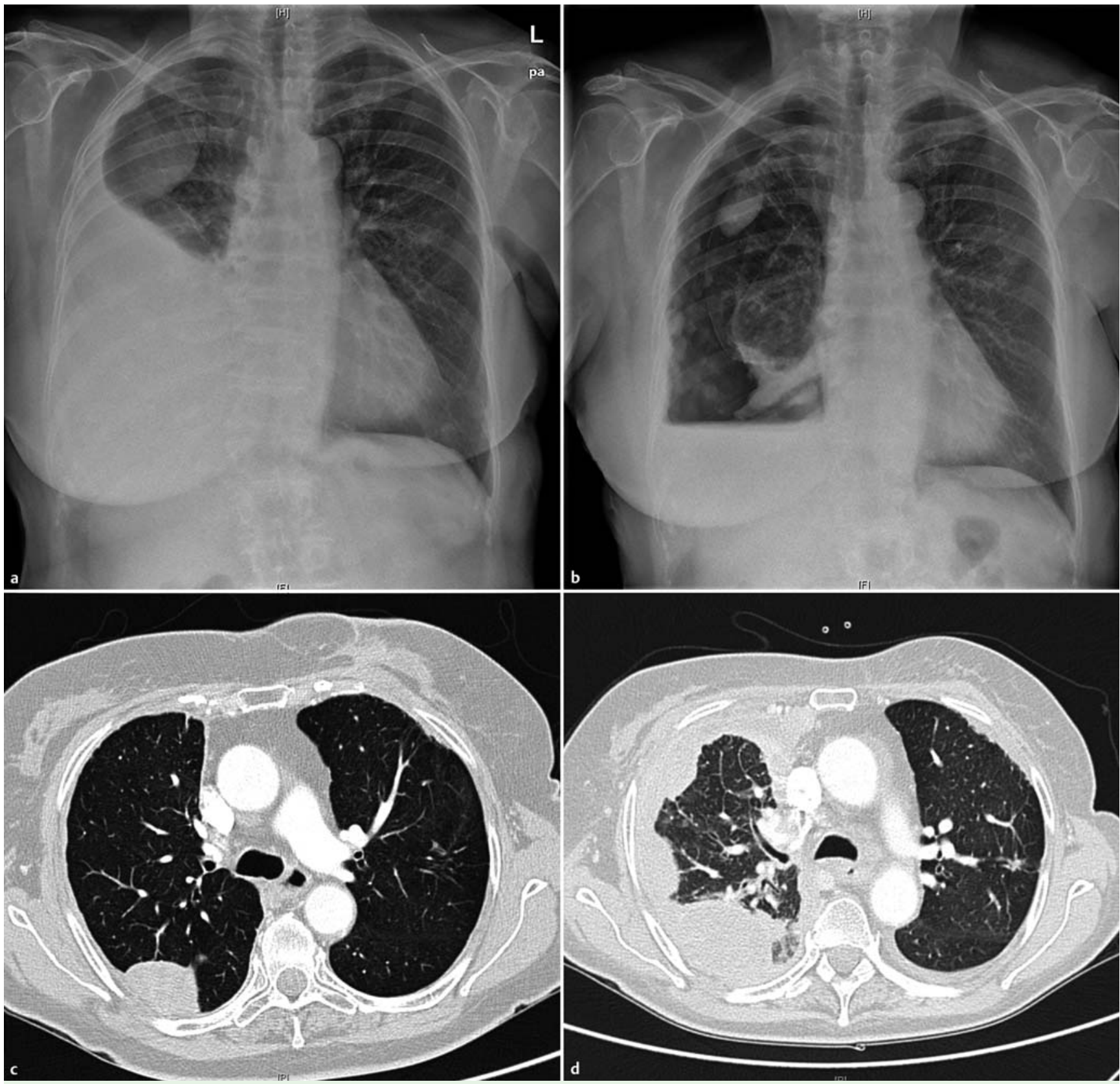

Abb.3 Der besondere Fall: Röntgenthorax einer 76-jährigen Patientin: a Pleuramesotheliom mit großem Erguss (p. a.-Übersichtsaufnahme) b Pleuramesotheliom und Erguss nach Anlage eines diagnostischen Pneumothorax (p. a.-Übersichtsaufnahme) c CT-Thorax der Patientin zeitgleich $\mathbf{d} 5$ Monate später.

\section{Ausblick}

$\nabla$

Asbeststaubexponierte bzw. ehemals exponierte Arbeiter müssen auf Grundlage der Gefährdungsbeurteilung eine arbeitsmedizinische Betreuung erhalten. Nach den „DGUV-lichen Grundsätzen der arbeitsmedizinischen Vorsorge“ werden Erst- und Nachuntersuchungen von nachgehenden Untersuchungen unterschieden. Erst- und Nachuntersuchungen werden während oder bei Beendigung der Tätigkeit durchgeführt. Nachgehende Untersuchungen stellen ein Angebot an die Versicherten dar, auch über das Ende des Beschäftigungsverhältnis hinaus arbeitsmedizinisch betreut zu werden. Diese nachgehenden Untersuchungen werden von der GVS (Gesundheitsvorsorge), ehemals ZAs (Zentrale Erfassungsstelle Asbestgefährdeter Arbeiter), einer Gemeinschaftseinrichtung der gesetzlichen Unfallversicherungsträger, organisiert. Maßgeblich hierfür ist der DGUV-liche Grundsatz G 1.2 „Asbestfaserhaltiger Staub“ $[31,56]$. Neben Arbeits- und Gesundheitsanamnese, körperlicher Untersuchung und Lungenfunktionsprüfung wird eine Röntgenaufnahme des Thorax angefertigt, bei unklaren Fällen wird zusätzlich eine Computertomografie bzw. ein HR-CT durchgeführt [57].

Die Früherkennung (Sekundärprävention) eines Pleuramesothelioms ist hinsichtlich des Therapieerfolges und der Krankheitsprognose von zentraler Bedeutung. Insbesondere bei schnell wachsenden Tumoren wie dem Pleuramesotheliom ist eine frühzeitige Detektion des Tumors besonders wünschenswert. Weltweit besteht jedoch ein großer Forschungsbedarf bzgl. effizienter und effektiver Methoden und Programmen zur Früherkennung. In Ergänzung der bildgebenden Verfahren wird in verschiedenen Forschungsgruppen versucht, Kandidaten-Biomarker zu detek- 
tieren bzw. in bestehenden Früherkennungsprogrammen zusätzlich zu validieren. Der Einsatz valider, nichtinvasiver Biomarker kann dabei in kürzeren Untersuchungsintervallen erfolgen, ohne zugleich die mögliche „untersuchungsinduzierte“ Strahlenbelastung zu erhöhen.

\section{Danksagung}

Die Autoren danken Herrn Univ.-Prof. Dr. A. Buchter für die freundliche Überlassung der Kasuistik und Herrn M. Velasco Garrido, Frau I. Richrath und Frau J. Fischer für ihre Unterstützung bei Literaturauswahl und Erstellung des Manuskripts.

\section{Interessenkonflikt}

Die Autoren geben an, dass kein Interessenkonflikt besteht.

\section{Literatur}

1 Europäisches Parlament, Rat der EU. Verordnung (EG) Nr. 1907/2006 des Europäischen Parlaments und des Rates vom 18. Dezember 2006 zur Registrierung, Bewertung, Zulassung und Beschränkung chemischer Stoffe (REACH), zur Schaffung einer Europäischen Agentur für chemische Stoffe, zur Änderung der Richtlinie 1999/45/EG und zur Aufhebung der Verordnung (EWG) Nr. 793/93 des Rates, der Verordnung (EG) Nr. 1488/94 der Kommission, der Richtlinie 76/769/EWG des Rates sowie der Richtlinien 91/155/EWG, 93/67/EWG, 93/105/EG und 2000/21/EG der Kommission. Artikel 67 in Verbindung mit Anhang XVII Nr. 6. Amtsbl EU L; 2006; 396: 1 -851. http://eur-lex.europa.eu/LexUriServ/LexUriServ.do?uri=0J:L:2006:396:0001:0001:DE: PDF

2 Virta RL. Asbestos. In: Interior USDot, Survey USG eds Mineral commodity summaries 2012. Reston: U. S. Government Printing Office; 2012: $22-23$

3 Peto J, Decarli A, La Vecchia C et al. The European mesothelioma epidemic. Br J Cancer 1999; 79: 666-672

4 Donaldson K, Murphy FA, Duffin $R$ et al. Asbestos, carbon nanotubes and the pleural mesothelium: a review of the hypothesis regarding the role of long fibre retention in the parietal pleura, inflammation and mesothelioma. Part Fibre Toxicol 2010; 7: 5

5 Ploenes T, Osei-Agyemang T, Nestle $U$ et al. [Malignant pleural mesothelioma]. Dtsch Med Wochenschr 2012; 137: 481-486

6 Rudd RM. Malignant mesothelioma. Br Med Bull 2010; 93: 105-123

7 Oberdörster G. Desposition, elimintation and effects of fibres in the respiratory tract of humans and animals. VDI Bericht 1991; 853: 17-38

8 Miserocchi G, Sancini G, Mantegazza F et al. Translocation pathways for inhaled asbestos fibers. Environ Health 2008: 7

9 Broaddus VC, Everitt JI, Black B et al. Non-neoplastic and neoplastic pleural endpoints following fiber exposure. J Toxicol Environ Health B Crit Rev 2011; 14: $153-178$

10 Manning $C B$, Vallyathan $V$, Mossman BT. Diseases caused by asbestos: mechanisms of injury and disease development. Int Immunopharmacol 2002; 2: $191-200$

11 Baur X, Clasen M, Fisseler-Eckhoff A et al. [Diagnostics and expert opinion of asbestos-induced occupational diseases]. Pneumologie 2011; 65: e1-47

12 NN BTS statement on malignant mesothelioma in the UK, 2007. Thorax 2007; 62: ii1 - ii 19

13 Merritt N, Blewett CJ, Miller JD et al. Survival after conservative (palliative) management of pleural malignant mesothelioma. J Surg Oncol 2001; 78: $171-174$

14 Ceresoli GL, Locati LD, Ferreri AJ et al. Therapeutic outcome according to histologic subtype in 121 patients with malignant pleural mesothelioma. Lung Cancer 2001; 34: 279-287

15 Rusch VW, Giroux D, Kennedy C et al. Initial Analysis of the International Association for the Study of Lung Cancer Mesothelioma Database. J Thorac Oncol 2012; 7: 1631-1639
16 Pinato DJ, Mauri FA, Ramakrishnan $R$ et al. Inflammation-based prognostic indices in malignant pleural mesothelioma. J Thorac Oncol 2012; 7: $587-594$

17 Raja S, Murthy SC, Mason DP. Malignant pleural mesothelioma. Curr Oncol Rep 2011; 13: 259-264

18 van Meerbeeck JP, Scherpereel A, Surmont VF et al. Malignant pleural mesothelioma: the standard of care and challenges for future management. Crit Rev Oncol Hematol 2011; 78: 92-111

19 Zahid I, Sharif S, Routledge T et al. What is the best way to diagnose and stage malignant pleural mesothelioma? Interact Cardiovasc Thorac Surg 2011; 12: 254-259

20 Kindler HL. Robust data: the essential foundation of a revised staging system for pleural mesothelioma. J Thorac Oncol 2012; 7: 1623-1624

21 Enderle G, Seidel H eds. Arbeitsmedizin. Fort- und Weiterbildung Kurs A - C. 1: ed. München: Urban und Fischer Verlag; 2004

22 Arendt M, Bauer HD, Blome $H$ et al. Faserjahre - Berufsgenossenschaftliche Hinweise zur Ermittlung der kummulativen AsbestfaserstaubDosis am Arbeitsplatz (Faserjahre) und Bearbeitungshinweise zur Berufskrankheit Nr. 4104 "Lungenkrebs oder Kehlkopfkrebs" durch Asbest. (BK-Report) 4. ed. St. Augustin: Hauptverband der gewerblichen Berufsgenossenschaften (HVBG); 2007

23 Mittmann $M$, Berger $H$, Neis $O$ et al. Arbeitsmedizinisches FortbildungsSymposium „Asbest: Eine Bilanz aus arbeitsmedizinischer Sicht“. Saarl Ärztebl 2008: 10-15

24 Baur X, Wegner R, Preisser A. Asbestbedingte Berufskrankheiten an Bord. Zbl Arbeitsmed 2012; 62: 244-247

25 Bundesregierung. Verordnung zum Schutz vor Gefahrstoffen (GefStoffV) Gefahrstoffverordnung vom 26. November 2010 (BGBl. I S 1643, 1644), die durch Artikel 2 des Gesetzes vom 28. Juli 2011 (BGBI I S. 1622) geändert worden ist. BGBl; 2011: 1622

26 Albracht G, Schwerdtfeger OA eds. Herausforderung Asbest. Wiesbaden: Universum Verlagsanstalt; 1991

27 Wagner JC, Sleggs CA, Marchand P. Diffuse pleural mesothelioma and asbestos exposure in the North Western Cape Province. Br J Ind Med 1960; 17: $260-271$

28 Kanarek MS. Mesothelioma from chrysotile asbestos: update. Ann Epidemiol 2011; 21: 688-697

29 Lenters V, Vermeulen R, Dogger S et al. A Meta-Analysis of Asbestos and Lung Cancer: Is Better Quality Exposure Assessment Associated with Steeper Slopes of the Exposure-Response Relationships? Environ Health Perspect 2011; 119: 1547 - 1555

30 Harth V, Korn M. Asbest. In: Landau K, Pressel G eds. Medizinisches Lexikon der beruflichen Belastungen und Gefährdungen. Wiesbaden: Universum Verlagsanstalt; 2009: 113-117

31 Deutsche Gesetzliche Unfallversicherung (DGUV). DGUV-Statistiken für die Praxis 2011. 2012: http://www.dguv.de/inhalt/zahlen/documents/dguvstatistiken2011d.pdf

32 Drechsel-Schlund C, Butz M, Haupt B et al. Asbestverursachte Berufskrankheiten in Deutschland - Entstehung und Prognose. Sankt Augustin: HVBG; 2002: http://www.dguv.de/inhalt/zahlen/documents/asbest.pdf

33 Price $B$, Ware $A$. Time trend of mesothelioma incidence in the United States and projection of future cases: an update based on SEER data for 1973 through 2005. Crit Rev Toxicol 2009; 39: 576-588

34 Hodgson JT, McElvenny DM, Darnton AJ et al. The expected burden of mesothelioma mortality in Great Britain from 2002 to 2050. Br J Cancer 2005; 92: 587-593

35 Grondin SC, Sugarbaker DJ. Malignant mesothelioma of the pleural space. Oncology (Williston Park) 1999; 13: 919-926; discussion 926 , 931-932

36 Delgermaa V, Takahashi K, Park EK et al. Global mesothelioma deaths reported to the World Health Organization between 1994 and 2008. Bull World Health Organ 2011; 89: 716 - 724 , 724A-724C

37 Bundesregierung. Berufskrankheiten-Verordnung vom 31.10.1997 (BGBl I S. 2623), zuletzt geändert durch Verordnung vom 11.6.2009. BGBl I; 2009: $1273-1276$

38 Price MJ, Darnton AJ, McElvenny DM et al. Simian virus 40 and mesothelioma in Great Britain. Occupational medicine (Oxford, England) 2007; 57 : 564-568

39 Merler E. A population-based study on radiotherapy as a risk-factor from malignant mesothelioma. In: The 9th International conference of the International Mesothelioma Interest Group 25-27 September. Amsterdam: 2008 
40 Karabin-Kehl B, Mittmann-Frank M, Berger $H$ et al. Arbeitsmedizinische Diagnostik bei Exposition gegenüber Nanopartikeln. Saarl Ärztebl 2011; 64: 17-26

41 Nagai H, Toyokuni S. Biopersistent fiber-induced inflammation and carcinogenesis: lessons learned from asbestos toward safety of fibrous nanomaterials. Arch Biochem Biophys 2010; 502: 1-7

42 Nagai H, Okazaki Y, Chew SH et al. Diameter and rigidity of multiwalled carbon nanotubes are critical factors in mesothelial injury and carcinogenesis. Proc Natl Acad Sci U S A 2011; 108: E1330 - 1338

43 Jaurand MC, Renier A, Daubriac J. Mesothelioma: Do asbestos and carbon nanotubes pose the same health risk? Part Fibre Toxicol 2009; 6: 16

44 Murphy FA, Poland CA, Duffin R et al. Length-dependent retention of carbon nanotubes in the pleural space of mice initiates sustained inflammation and progressive fibrosis on the parietal pleura. Am J Pathol 2011; 178: 2587-2600

45 Schinwald A, Murphy FA, Prina-Mello A et al. The threshold length for fiber-induced acute pleural inflammation: shedding light on the early events in asbestos-induced mesothelioma. Toxicol Sci 2012; 128: $461-470$

46 Poland CA, Duffin R, Kinloch I et al. Carbon nanotubes introduced into the abdominal cavity of mice show asbestos-like pathogenicity in a pilot study. Nature Nanotechnology 2008; 3: 423-428

47 Takagi A, Hirose A, Futakuchi M et al. Dose-dependent mesothelioma induction by intraperitoneal administration of multi-wall carbon nanotubes in p53 heterozygous mice. Cancer Sci 2012; 103: 1440 1444

48 Takagi A, Hirose A, Nishimura T et al. Induction of mesothelioma in p53 + /- mouse by intraperitoneal application of multi-wall carbon nanotube. J Toxicol Sci 2008; 33: 105-116
49 Sakamoto $Y$, Nakae D, Fukumori $N$ et al. Induction of mesothelioma by a single intrascrotal administration of multi-wall carbon nanotube in intact male Fischer 344 rats. J Toxicol Sci 2009; 34: 65 - 76

50 Pacurari M, Castranova V, Vallyathan V. Single- and multi-wall carbon nanotubes versus asbestos: are the carbon nanotubes a new health risk to humans? J Toxicol Environ Health A 2010; 73: 378-395

51 Ogasawara $Y$, Umezu N, Ishii $K$. [DNA damage in human pleural mesothelial cells induced by exposure to carbon nanotubes]. Nihon Eiseigaku Zasshi 2012; 67: 76-83

52 Varga C, Szendi K. Carbon nanotubes induce granulomas but not mesotheliomas. In Vivo 2010; 24: 153-156

53 Aschberger K, Johnston HJ, Stone $V$ et al. Review of carbon nanotubes toxicity and exposure-appraisal of human health risk assessment based on open literature. Crit Rev Toxicol 2010; 40: 759-790

54 Stella GM. Carbon nanotubes and pleural damage: perspectives of nanosafety in the light of asbestos experience. Biointerphases 2011; 6: P1 - 17

55 Ausschuss für Gefahrstoffe (AGS). Verordnung zur Neufassung der Gefahrstoffverordnung und zur Änderung sprengstoffrechtlicher Verordnungen vom 26.11.2010. BGBI Teil I; 2010: 1643-1692

56 Deutsche Gesetzliche Unfallversicherung (DGUV). ed. DGUV Grundsätze für arbeitsmedizinische Vorsorgeuntersuchungen. 5. ed. Stuttgart: Gentner Verlag; 2010

57 BGETEM (Energie Textil Elektro Medienerzeugnisse). Gesundheitsvorsorge (GVS) - vormals ZAs.http://gvs.bgetem.de

58 Wittekind C, Meyer HJ eds. TNM Klassifikation maligner Tumoren. Weinheim: Wiley-VCH; 2010 